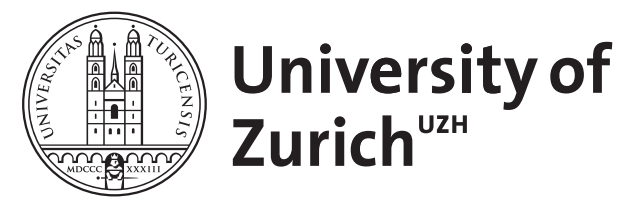

\title{
Microlensing masses via photon bunching
}

\author{
Saha, Prasenjit
}

\begin{abstract}
In microlensing of a Galactic star by a brown dwarf or other compact object, the amplified image really consists of two unresolved images with slightly different light traveltimes. The difference (of the order of a microsecond) is GM/c3 times a dimensionless factor depending on the total magnification. Since magnification is well measured in microlensing events, a single time-delay measurement would provide the mass of the lens, without degeneracies. The challenge is to find an observable that varies on submicrosecond time-scales. This paper notes that the narrow-band intensity of the unresolved image pair will show photon bunching (the Hanbury Brown and Twiss effect), and argues that the lensed intensity will have an autocorrelation peak at the lensing time delay. The ultrafast photon-counting technology needed for this type of measurement exists, but the photon numbers required to give sufficient signal-tonoise appear infeasible at present. Preliminary estimates suggest time-delayed photon bunching may be measurable for lensed early-type main-sequence stars at $10 \mathrm{kpc}$, with the help of $30 \mathrm{~m}$-class telescopes.
\end{abstract}

DOI: https://doi.org/10.1093/mnras/stz1208

Posted at the Zurich Open Repository and Archive, University of Zurich

ZORA URL: https://doi.org/10.5167/uzh-176190

Journal Article

Published Version

Originally published at:

Saha, Prasenjit (2019). Microlensing masses via photon bunching. Monthly Notices of the Royal Astronomical Society, 486(4):5400-5404.

DOI: https://doi.org/10.1093/mnras/stz1208 


\title{
Microlensing masses via photon bunching
}

\author{
Prasenjit Saha ${ }^{\circledR \star}$ \\ Physik-Institut, University of Zurich, Winterthurerstrasse 190, CH-8057 Zurich, Switzerland
}

Accepted 2019 April 29. Received 2019 April 24; in original form 2019 February 20

\begin{abstract}
In microlensing of a Galactic star by a brown dwarf or other compact object, the amplified image really consists of two unresolved images with slightly different light traveltimes. The difference (of the order of a microsecond) is $G M / c^{3}$ times a dimensionless factor depending on the total magnification. Since magnification is well measured in microlensing events, a single time-delay measurement would provide the mass of the lens, without degeneracies. The challenge is to find an observable that varies on submicrosecond time-scales. This paper notes that the narrow-band intensity of the unresolved image pair will show photon bunching (the Hanbury Brown and Twiss effect), and argues that the lensed intensity will have an autocorrelation peak at the lensing time delay. The ultrafast photon-counting technology needed for this type of measurement exists, but the photon numbers required to give sufficient signal-to-noise appear infeasible at present. Preliminary estimates suggest time-delayed photon bunching may be measurable for lensed early-type main-sequence stars at $\sim 10 \mathrm{kpc}$, with the help of $30 \mathrm{~m}$-class telescopes.
\end{abstract}

Key words: gravitational lensing: micro - techniques: interferometric.

\section{INTRODUCTION}

In Galactic microlensing, there are two lensed images (more if the lens is binary), but there is no prospect of resolving them. Everything has to be inferred from a point image.

The principal observable is (i) the time-dependent brightness amplification (or light curve). Link (1936, 1937) and independently Einstein (1936) discussed it long before it became feasible to observe, with strikingly contrasting views on whether it could ever be observed. Later but still in the dream-time of gravitational lensing, Refsdal (1966a) drew attention to two more observables: (ii) parallax, which in the context of microlensing refers to the dependence of the light curve on observer location and (iii) apparent proper motion of the image. Parallax depends on the spatial scale of the lens, while proper motion reveals its angular scale. Combining parallax and proper motion with the light curve yields the lens mass and distance. For parallax, Refsdal envisioned a spacecraft elsewhere in the Solar system, an idea revived more recently (e.g. Boutreux \& Gould 1996). For proper motions, Refsdal assumed that both source and lens would be visible stars. An alternative proposal, for when the lens is dark, is to monitor the lensed-image centroid for proper motion (e.g. Boden, Shao \& Van Buren 1998). VallsGabaud (1995, 1998) predicted a further observable: (iv) chromatic amplification, meaning colour, spectral, and polarization changes in the image due to variations of these across the face of the source star. Given a good model for the stellar atmosphere, chromatic

^E-mail: psaha@physik.uzh.ch amplification becomes in effect a surrogate for proper motion. Various observables come together beautifully in Gould et al. (2009), where chromatic amplification gives the angular scale, while the parallax is large enough to measure from different ground locations, and combined with the main light curve they supply the lens mass. But these cases are exceptional; as a rule, mass measurement in microlensing involves parameter degeneracies.

This paper will consider yet another possible observable in an unresolved microlensed image. This is the lensing time delay, or the difference in light traveltime between the two lensed images. The formula for it is derived in Section 2 and comes to

$\Delta t_{\text {lens }}=\frac{2 G M}{c^{3}}\left(x^{2}-1 / x^{2}+4 \ln x\right)$,

where

$x^{4}=\frac{A+1}{A-1}$

and $A$ is the brightness amplification or total magnification. Since $A$ at any stage of a microlensing event is known accurately from the light curve, a single measurement of $\Delta t_{\text {lens }}$ would automatically measure the mass.

Time-delay measurements are an active area for galaxy and cluster lenses at cosmological distances (e.g. Rodney et al. 2016). There is also research on modelling the mass distributions of the lenses (as the point-mass approximation is not applicable to galaxies and cluster lenses) to infer cosmological parameters (e.g. Sereno \& Paraficz 2014) or mass substructures (Mohammed, Saha \& Liesenborgs 2015). And long before the first time-delay measurements, Refsdal (1964a, 1966b) was already advocating 
exploiting time-varying sources to measure time delays from galaxy lenses in order to measure cosmological parameters. Why then did he not propose time-delay measurements in his microlensing paper (Refsdal 1966a)? We can guess the reason: the scale of $\Delta t_{\text {lens }}$ is utterly different - microseconds for brown-dwarf lenses versus days to years for galaxy or cluster lenses - and stars are not known to have intrinsic brightness variations on submicrosecond scales. Measuring microlensing time delays seems hopeless.

Yet perhaps not. Section 3 below will argue that fluctuations inherent in incoherent light (wave noise) could be used to advantage. The idea is to measure the brightness fluctuations, at any one stage of the microlensing event, with nanosecond time resolution. The photon statistics will then not be quite Poisson, but will show correlations. In particular, the autocorrelation of the photon arrival times will show the following features:

(i) First, there will be a peak at zero. This is the well-known phenomenon of photon bunching.

(ii) Also expected - and this is the main prediction of this paperare two smaller peaks at $\pm \Delta t_{\text {lens }}$, corresponding to the lensing time delay, or the difference in light traveltime between the two lensed images.

Section 4 estimates the number of photons that would be needed to measure the secondary peaks in the intensity autocorrelation, assuming the effect is present. If nearby bright stars were microlensed, the autocorrelation peaks would be easy to measure. For stars at $\sim 10 \mathrm{kpc}$, which is where most microlensed sources are, the necessary signal-to-noise appears unachievable at present. But upcoming developments (30 m telescopes, subnanosecond photoncounting arrays) could make the effect accessible for the brightest microlensed events.

\section{MICROLENSING TIME DELAYS}

To derive expression (1) for the time delay in microlensing, let us consider the arrival-time surface (see e.g. Blandford \& Narayan 1986). The arrival time, up to an additive constant, of a virtual photon coming from the direction $\theta$ but having originated at a source in the direction $\beta$ is

$c t=\frac{D_{\mathrm{L}} D_{\mathrm{S}}}{2 D_{\mathrm{LS}}}(\beta-\theta)^{2}-\frac{4 G M}{c^{2}} \ln \theta$,

where $D_{\mathrm{L}}, D_{\mathrm{S}}$, and $D_{\mathrm{LS}}$ are, respectively, the distances to the lens, source, and from lens to source. In terms of the angular Einstein radius

$\theta_{\mathrm{E}}^{2} \equiv \frac{4 G M}{c^{2}} \frac{D_{\mathrm{LS}}}{D_{\mathrm{S}} D_{\mathrm{L}}}$,

the arrival time takes a cleaner form:

$c t=\frac{4 G M}{c^{2}}\left(\frac{(\beta-\theta)^{2}}{2 \theta_{\mathrm{E}}^{2}}-\ln \theta\right)$.

Actual photons take paths for which the arrival time is stationary, or $\mathrm{d} t / \mathrm{d} \theta=0$. This condition gives the usual lens equation

$\beta=\theta-\theta_{\mathrm{E}}^{2} / \theta$.

Solutions of the lens equation can be conveniently written in terms of a new variable $x$ as

$\theta=x \theta_{\mathrm{E}}$ and $\theta=-\theta_{\mathrm{E}} / x$.

The source position corresponding to both images is

$\beta=(x-1 / x) \theta_{\mathrm{E}}$.
Substituting the image and source positions $(7,8)$ into the arrival time (5) gives the value for each image. The difference between the arrival times of the two images then simplifies to expression (1) for the time delay.

In order to relate $x$ to the brightness amplification, recall the well-known expression

$$
\left(1-\frac{\theta_{\mathrm{E}}^{4}}{\theta^{4}}\right)^{-1}
$$

for the magnification due to a point lens. The absolute magnification at the two images comes to

$A_{1}=\frac{x^{4}}{x^{4}-1}$ and $A_{2}=\frac{1}{x^{4}-1}$

and the sum of these relates $x$ to the total magnification (equation 2).

The preceding applies only to a point source lensed by a point lens. For a realistic source, even an unresolved source, it is necessary to consider the effect of finite size. From expressions (1) for the time delay and (8) for the source position, it follows that

$\frac{\mathrm{d}}{\mathrm{d} \beta} \Delta t_{\mathrm{lens}}=\frac{4 G M}{c^{3}} \frac{x+1 / x}{\theta_{\mathrm{E}}}$,

indicating that

$\frac{\Delta \Delta t_{\text {lens }}}{\Delta t_{\text {lens }}} \sim \frac{\Delta \beta}{\theta_{\mathrm{E}}}$,

where $\Delta \Delta t_{\text {lens }}$ stands for the spread in time delays across the source. As an example, consider the configuration

$$
\begin{aligned}
& D_{\mathrm{L}}=4 \mathrm{kpc} \quad D_{\mathrm{S}}=8 \mathrm{kpc} \\
& M=0.08 \mathrm{M}_{\odot} \quad \beta=\frac{1}{2} \theta_{\mathrm{E}},
\end{aligned}
$$

which would be typical of microlensing events. The projected Einstein radius will be $D_{\mathrm{L}} \theta_{\mathrm{E}}=1.1$ au at the lens, and $D_{\mathrm{S}} \theta_{\mathrm{E}}=2.2 \mathrm{au}$ at the source. If the source is Sun-sized, it will be much smaller than the projected Einstein radius, and the point-lens approximation is reasonable. Furthermore, $\Delta \Delta t_{\text {lens }} \ll \Delta t_{\text {lens }}$. The spread in time delays will, however, be orders of magnitude larger than the $1 / v$ of light. This is the reason lensed images do not produce interference fringes on the ground (cf. Refsdal 1964b; Press 1996). Fig. 1 shows a numerical computation of arrival times from an extended source. We see that for a Sun-sized source in the lensing configuration (13), $\Delta t_{\text {lens }} \simeq 1.6 \times 10^{-6} \mathrm{~s}$ and $\Delta \Delta t_{\text {lens }} \sim 10^{-8} \mathrm{~s}$.

We conclude that microlensing time delays could be measured if the light from the source has fluctuations that are faster than the microsecond scale, but not so fast that they average out within 10 ns. The following section will argue that the desired fluctuations may be found in photon bunching.

\section{PHOTON BUNCHING}

Photon bunching, or the HBT effect (named after the pioneering experiments of Hanbury Brown \& Twiss 1956), is a quantum-optical phenomenon, but can be studied semiclassically by first considering a complex wave and then interpreting the intensity of the wave as proportional to the probability of detecting photons. ${ }^{1}$

Accordingly, let us consider the wave (a component of the electromagnetic field) for starlight in a narrow-band $S(v)$. The

\footnotetext{
${ }^{1}$ This was first shown by Sudarshan (1963) and is nowadays called the
} optical equivalence theorem. 

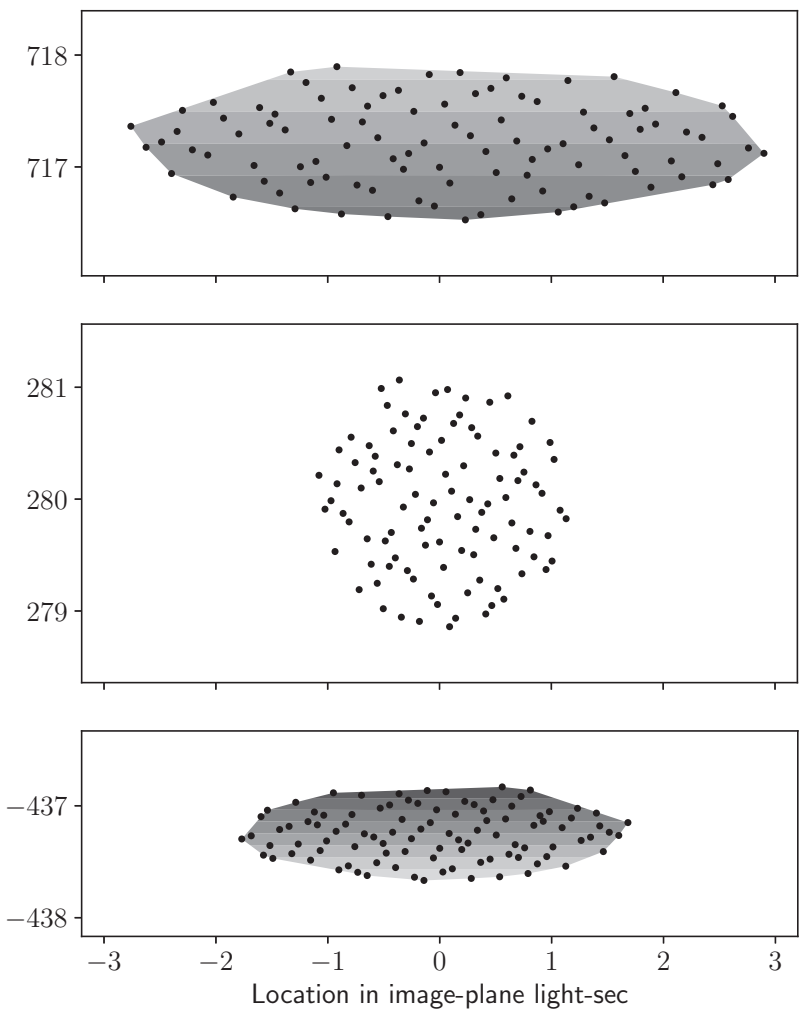

Figure 1. A representation of the lensed images and arrival times for a solarsized source in the lensing configuration (13). The middle panel shows the source while the other two panels show the lensed images. (The lens is at the origin.) The dots represent a quasi-random sample of 100 zones on the source. The grey scale indicates the spread of arrival times within each image (darker for later arrival). The spread is $\Delta \Delta t_{\text {lens }}=5 \mathrm{~ns}$ for the earlier image, and $8 \mathrm{~ns}$ for the lower image. The systemic difference between the images is much more, with the lower image arriving $\Delta t_{\text {lens }}=1584 \mathrm{~ns}$ later on average.

complex wave will then be

$E(t)=\int \mathrm{e}^{2 \pi \mathrm{i} v t} S(v) \mathrm{d} v$,

and the corresponding intensity will be

$I(t) \propto|E(t)|^{2}$.

If $S(v)$ is a delta function, $E(t)$ will just revolve in the complex plane at a constant rate and $I(t)$ will be a constant. But if $S(v)$ has a finite width, $E(t)$ will a superposition of contributions with random phases in the complex plane, making it like the endpoint of a random walk. Thus the probability distribution for $E(t)$ will be Gaussian in the complex plane, and the intensity will have an exponential distribution. The larger the frequency spread in $S(v)$ the more quickly $E(t)$ and $I(t)$ will sample their respective probability distributions. In particular, if $S(v)$ is a Lorentzian

$S(v) \propto \frac{1}{1+\left(2 \pi \Delta \tau\left(v-v_{0}\right)\right)^{2}}$,

the time-scale for the field and intensity to change will be $\Delta \tau$. This is known as the coherence time. ${ }^{2}$ For a nanometre filter, the

\footnotetext{
${ }^{2}$ The precise definition of coherence time varies in the literature. This work
} follows $\Delta \tau_{2}$ from Mandel \& Wolf (1962).
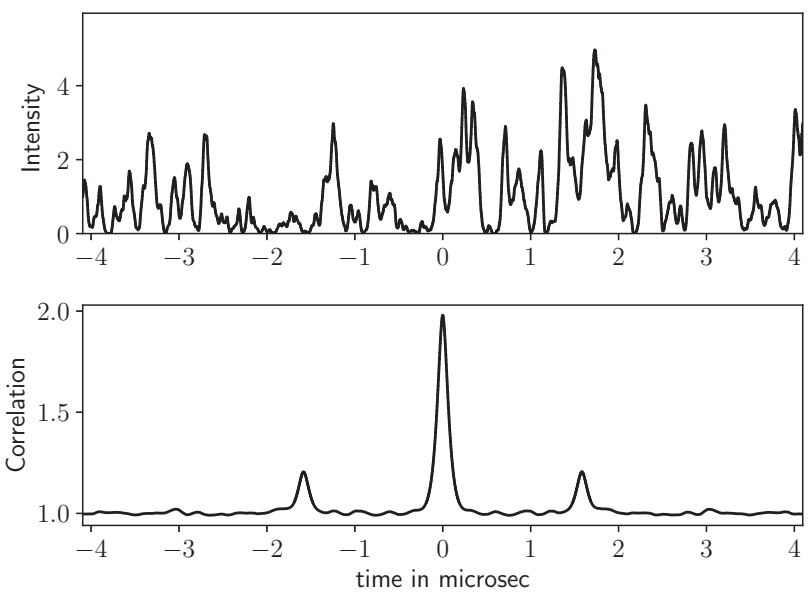

Figure 2. A simulation of lensed intensity $I(t)$ and autocorrelation $1+g(\tau)$ from the system shown in Fig. 1.

coherence time is roughly $1 \mathrm{ps}$. The intensity autocorrelation

$1+g(\tau) \equiv \frac{\langle I(t) I(t+\tau)\rangle}{\langle I(t)\rangle^{2}}$

will have $g(0)=2$ falling over a time $\Delta \tau$ to $g(\tau)=1$. This is the standard HBT effect.

Next we consider the effect of magnification by lensing. For an image magnified by say $A_{1}$, the intensity $I(t)$ will get multiplied by $A_{1}$. The complex wave $E(t)$ must get multiplied by $\sqrt{A_{1}}$ times a phase factor. The multiplication by $\sqrt{A_{1}}$ seems a little mysterious, but can be understood as follows. Recall that lensing magnification is the result of light rays, which would not have reached the observer without lensing, being deflected by the lens towards the observer. The corresponding complex waves add, but since they have random phases they add in random-walk fashion, which on average produces a factor of $\sqrt{A_{1}}$.

Now consider a superposition of the waves from two lensed images. The intensity will be

$I(t)=\left|\sqrt{A_{1}} E(t)+\sqrt{A_{2}} E\left(t+\Delta t_{\text {lens }}\right)\right|^{2}$.

The waves $E(t)$ and $E\left(t+\Delta t_{\text {lens }}\right)$ are uncorrelated in phase, so there is no interference. The intensities, however, may be correlated. The autocorrelation (17) is expected to show secondary peaks of height $A_{1} A_{2} /\left(A_{1}+A_{2}\right)^{2}$, or

$g\left( \pm \Delta t_{\text {lens }}\right)=\frac{A^{2}-1}{4 A^{2}}$,

in addition to the main peak of $g(0)=1$.

Fig. 2 shows a simulation of lensed intensity and autocorrelation, obtained as follows.

(i) Each of the 100 zones in Fig. 1 is taken to emit a wave $E(t)$ having a Lorentzian spectral profile with $\Delta=10^{-8} \mathrm{~s}$. The central frequency $v_{0}$ makes no difference to the intensity and is set to zero, but the wave from each zone is given a random initial phase. The simulated time duration is $1 \mathrm{~ms}$, of which only $\simeq 8 \mu \mathrm{s}$ is shown in the figure.

(ii) Each wave is lensed in the same lensing configuration as in Fig. 1. That is, each wave is delayed by the appropriate amount and multiplied by $\sqrt{A_{1}}$ or $\sqrt{A_{2}}$.

(iii) All the waves are added, and the intensity computed. 
(iv) Finally the intensity is autocorrelated. Expected are a central peak rising to 2, and (in the assumed lensing configuration) secondary peaks at $\pm 1.6 \mathrm{~ms}$, each rising to 1.2 .

Note that there is no shot noise in Fig. 2. The intensity fluctuations are just a property of narrow-band light. They are well known in radio astronomy as wave noise (Radhakrishnan 1999). Photon counts will follow a Poisson distribution with the intensity varying over a time-scale of $\Delta \tau$. The result is photon bunching. The term 'super-Poisson noise' is also used.

The secondary peaks in the autocorrelation are very interesting but are the arguments and simulation valid? There are at least three possible concerns.

(i) First, it is surprising that the intensity autocorrelation appears not to depend on $v_{0}$, only on $\Delta v$.

(ii) Secondly, there is the assumption that the source can be expressed as the sum of small zones. The simulation is not sensitive to the number of zones. On the other hand, the sample points representing zones are $\sim 0.5$ light-sec part, which is an order of magnitude larger than the Fresnel-zone length $\sqrt{\lambda D_{\mathrm{S}}}$ associated with diffraction. Actually, diffraction effects are possible on even smaller scales, though only near lensing caustics (e.g. Zabel \& Peterson 2003), which does not apply to most microlensing events.

(iii) Thirdly, the factors of $\sqrt{A_{1}}$ and $\sqrt{A_{2}}$ in the total intensity (18) would come with arbitrary phases, but again, these would not destroy the autocorrelation.

A laboratory experiment to test for time-delayed photon bunching is desirable. A suitable variant of the experiments by Dravins, Lagadec \& Nuñez (2015) and Tan, Chan \& Kurtsiefer (2016) could do such a test.

\section{SIGNAL-TO-NOISE CONSIDERATIONS}

Assuming time-delayed photon bunching is real, identifying the most promising events in advance would not be a problem. Although Galactic microlensing events are rare, with at most a few stars per million being lensed at any given time (Sumi et al. 2013), an earlywarning system (such as in Udalski, Szymański \& Szymański 2015) would provide the expected image brightness and magnification. The challenge would be getting sufficient signal-to-noise to measure the time-delayed photon bunching on even the best candidates.

The basic set-up and signal-to-noise considerations would be similar to those in recent work on intensity interferometry (Pilyavsky et al. 2017; Lai et al. 2018; Weiss, Rupert \& Horch 2018). The starlight is filtered to a very narrow wavelength band, to increase the coherence time $\Delta \tau$, and then photons are counted with a time resolution $\Delta t$. The favoured detector technology is single-photon avalanche photodiodes. Multiple photodiodes, each devoted to one narrow wavelength range, can be used in parallel, thus having multiple photon-counting channels.

In intensity interferometry, there are two (or more) telescopes whose photon counts are cross-correlated, whereas for microlensing only one telescope is required - but a bigger one, because the targets are fainter. An additional requirement in microlensing is to have the coherence time $\Delta \tau$ comparable to or longer than the time-delay spread $\Delta \Delta t_{\text {lens }}$. The latter condition could be checked in advance, since an approximate source size $\Delta \beta$ would be available for an ongoing microlensing event, providing ballpark estimates for $\Delta t_{\text {lens }}$ and $\Delta \Delta t_{\text {lens }}$.

The signal-to-noise ratio (SNR) can be estimated by adapting an argument from intensity interferometry, as follows. Let $r$ be the rate of photons arriving per unit collecting area in some narrow spectral band, and let $\Delta \tau \sim 1 / \Delta v$ be the coherence time corresponding to that spectral band. If the telescope has unit collecting area and perfect detection efficiency, the number of photons per coherence time will be $r \Delta \tau$. Now consider two time bins, each of duration $\Delta t$ (the instrumental time resolution), but separated by $\Delta t_{\text {lens }}$.

(i) Each time bin will contain $\Delta t / \Delta \tau$ time slices, during which the light is coherent. In a pair of coherent time slices $\Delta t_{\text {lens }}$ apart, there will be $g\left(\Delta t_{\text {lens }}\right) \times(r \Delta \tau)^{2}$ pairs of HBT-correlated photons. Hence, there will be $g\left(\Delta t_{\text {lens }}\right) \times r^{2} \Delta \tau \Delta t$ HBT-correlation events per time bin. This is the signal.

(ii) Meanwhile, in the same time bins, there will be $(r \Delta t)^{2}$ pairs of photons correlated by chance. This number is the background, and $r \Delta t$ is the corresponding noise.

The SNR per time bin $\Delta t$ is thus $g\left(\Delta t_{\text {lens }}\right) \times r \Delta \tau$. This applies to unit collecting area and perfect detectors. If we have collecting area $A$ and photon-detection efficiency $\gamma$, these factors just multiply $r$. Concerning the spectral bandpass, narrowing it reduces $r$ but increases $\Delta \tau$ by the same factor, and (remarkably) leaves the SNR per time bin unaffected. Hence, it is advantageous to have many narrow spectral channels. For $N$ channels, the SNR gets multiplied by $\sqrt{N}$. Similarly, if the total observing time is $T$, the SNR will be multiplied by $\sqrt{T / \Delta t}$. The result is

$\mathrm{SNR} \sim g\left(\Delta t_{\text {lens }}\right) \times \gamma A r \Delta \tau\left(\frac{N T}{\Delta t}\right)^{1 / 2}$

provided $\Delta t \gtrsim \Delta \tau$. The SNR improves as the time resolution gets smaller, until it becomes comparable to the coherence time. Lowering $\Delta t$ still further does not help, as super-Poisson noise takes over (Malvimat, Wucknitz \& Saha 2014). The factor $g\left(\Delta t_{\text {lens }}\right)$ will be less than one, but roughly compensating for that is the increase in $r$ from lensing amplification. So it is reasonable to consider $(\gamma A) \times(r \Delta \tau) \times \sqrt{N T / \Delta t}$ without lensing.

To reach a reasonable SNR in one night, one would need to achieve SNR $\sim 1$ in $T=10^{3} \mathrm{~s}$. If $N=10$ channels each with $\Delta t=10^{-8} \mathrm{~s}$ are installed, $\sqrt{N T / \Delta t} \sim 10^{6}$. Hence, one needs $(\gamma A) \times(r \Delta \tau) \sim 10^{-6}$ at least. The next generation of extremely large telescopes and highly efficient detectors could offer $\gamma A \sim$ $10^{3} \mathrm{~m}^{2}$ at best. This suggests that sources down to $\Delta \tau \sim 10^{-9} \mathrm{~m}^{-2}$ would be plausible targets.

The photon flux per coherence time $r \Delta \tau$ is simply the spectral flux density divided by energy and integrated over the source. Fig. 3 shows some example curves of $r \Delta \tau$. A blackbody disc of radius $\mathrm{R}_{\odot}$ and $T=5800 \mathrm{~K}$ viewed from $1 \mathrm{pc}$ is labelled 'Sun'. A disc of radius $0.2 \mathrm{R}_{\odot}$ and $T=3100 \mathrm{~K}$ viewed from the same distance is labelled 'Barnard's star'. Similarly, the label 'Sirius' corresponds to $1.7 \mathrm{R}_{\odot}$ and $9900 \mathrm{~K}$ from $1 \mathrm{pc}$, and 'Achernar' to $9 \mathrm{R}_{\odot}$ and $T=15000 \mathrm{~K}$ from $1 \mathrm{pc}$. From such a short distance, any of these would be a plausible candidate. But if they are located at $\sim 10 \mathrm{kpc}$, the value of $r \Delta \tau$ falls by a factor of $10^{8}$. This leaves only 'Achernar' as a plausible candidate.

The above suggests that photon bunching would be measurable in $\mathrm{O}$ or $\mathrm{B}$ main-sequence stars at $\sim 10 \mathrm{kpc}$. (Giant or supergiant stars of similar brightness are unlikely to be useful, because $\Delta \beta$ will become comparable to $\theta_{\mathrm{E}}$, washing out the time-delay peaks.) Microlensed early-type stars must be exceedingly rare, and it is not clear that any have been observed yet. Nataf, Stanek \& Bakos (2009) show some candidate light curves in their fig. 5, which may have been such, if they were indeed microlensing events.

Plans for intensity interferometers include proposals to create light buckets as large as $10^{4} \mathrm{~m}^{2}$ by attaching additional detectors to 


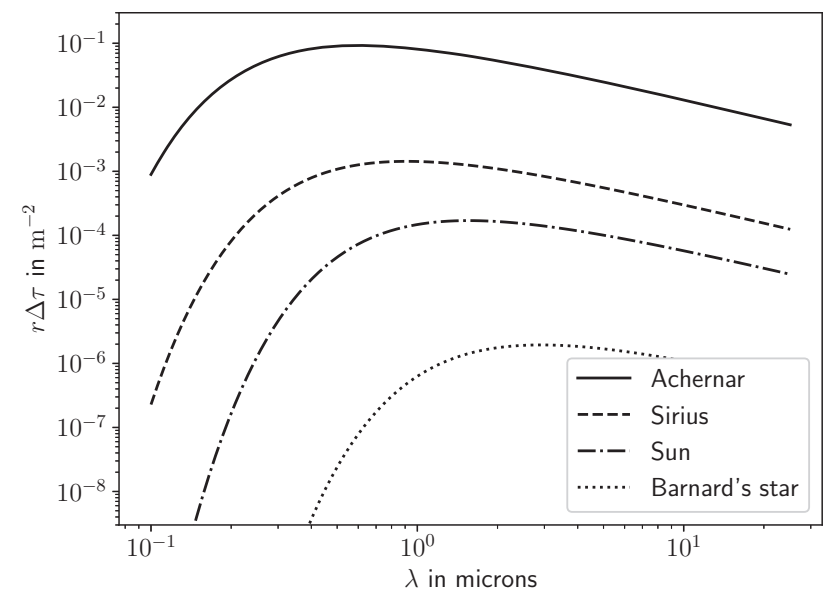

Figure 3. Curves of $r \Delta \tau$ for four different values of radius and surface temperature (corresponding approximately to the named stars) but viewed from 1 parsec in all cases.

air Cerenkov telescopes (Dravins 2016). The mirrors involved are not optical quality (hence the huge areas). These sacrifice image quality but remain adequate for collecting light from bright stars. Microlensing surveys, however, need to target crowded fields of stars, and to count photons from a single star in a crowded field, good image quality is essential. Hence a general-purpose telescope cannot be substituted, and there is no way of increasing $A$ in equation (20) in the near future. Any hope for increased sensitivity would lie in increasing the number of spectral channels $N$. Small arrays of single-photon avalanche photodiodes have been tested (e.g. Tosi et al. 2014), and perhaps much larger arrays may be possible in the future. Another class of detectors with prospects for manychannel photon counting is superconducting nanowires (e.g. Verma et al. 2015).

\section{REFERENCES}

Blandford R., Narayan R., 1986, ApJ, 310, 568

Boden A. F., Shao M., Van Buren D., 1998, ApJ, 502, 538

Boutreux T., Gould A., 1996, ApJ, 462, 705

Dravins D., 2016, in Fabien M., Michelle J. C.-E., Peter G. T., eds, Proc. SPIE

Conf. Ser. Vol. 9907, Optical and Infrared Interferometry and Imaging

V. SPIE, Bellingham, p. 99070M
Dravins D., Lagadec T., Nuñez P. D., 2015, A\&A, 580, A99

Einstein A., 1936, Science, 84, 506

Gould A. et al., 2009, ApJ, 698, L147

Hanbury Brown R., Twiss R. Q., 1956, Nature, 177, 27

Lai O. et al., 2018, in Michelle J. C.-E., Peter G. T., Antoine M., eds, Proc. SPIE Conf. Ser. Vol. 10701, Optical and Infrared Interferometry and Imaging VI. SPIE, Bellingham, p. 1070121

Link F., 1936, C. R. Acad. Sci., 202, 917

Link F., 1937, Bull. Astron., 10, 73

Malvimat V., Wucknitz O., Saha P., 2014, MNRAS, 437, 798

Mandel L., Wolf E., 1962, Proc. Phys. Soc., 80, 894

Mohammed I., Saha P., Liesenborgs J., 2015, PASJ, 67, 21

Nataf D. M., Stanek K. Z., Bakos G. A., 2009, Acta Astron., 59, 255

Pilyavsky G., Mauskopf P., Smith N., Schroeder E., Sinclair A., van Belle G. T., Hinkel N., Scowen P., 2017, MNRAS, 467, 3048

Press W. H., 1996, in Kochanek C. S., Hewitt J. N., eds, Proc. IAU Symp. 173, Astrophysical Applications of Gravitational Lensing. Kluwer, Dordrecht, p. 407

Radhakrishnan V., 1999, in Taylor G. B., Carilli C. L., Perley R. A., eds, ASP Conf. Ser. Vol. 180, Synthesis Imaging in Radio Astronomy II. Astron. Soc. Pac., San Francisco, p. 671

Refsdal S., 1964a, MNRAS, 128, 307

Refsdal S., 1964b, MNRAS, 128, 295

Refsdal S., 1966a, MNRAS, 134, 315

Refsdal S., 1966b, MNRAS, 132, 101

Rodney S. A. et al., 2016, ApJ, 820, 50

Sereno M., Paraficz D., 2014, MNRAS, 437, 600

Sudarshan E. C., 1963, Phys. Rev. Lett., 10, 277

Sumi T. et al., 2013, ApJ, 778, 150

Tan P. K., Chan A. H., Kurtsiefer C., 2016, MNRAS, 457, 4291

Tosi A. et al., 2014, in Mark A. I., ed., Proc. SPIE Conf. Ser. Vol. 9114, Advanced Photon Counting Techniques VIII. SPIE, Bellingham, p. 91140C

Udalski A., Szymański M. K., Szymański G., 2015, Acta Astron., 65, 1

Valls-Gabaud D., 1995, in Mücket J. P., Gottloeber S., Müller V., eds, Large Scale Structure in the Universe, World Scientific, Singapore, p. 326

Valls-Gabaud D., 1998, MNRAS, 294, 747

Verma V. B. et al., 2015, Opt. Express, 23, 33792

Weiss S. A., Rupert J. D., Horch E. P., 2018, in Michelle J. C.-E., Peter G. T., Antoine M., eds, Proc. SPIE Conf. Ser. Vol. 10701, Optical and Infrared Interferometry and Imaging VI. SPIE, Bellingham, p. 107010X

Zabel S. A., Peterson J. B., 2003, ApJ, 594, 456

This paper has been typeset from a $\mathrm{T}_{\mathrm{E}} \mathrm{X} / \mathrm{LT} \mathrm{E} \mathrm{X}$ file prepared by the author. 